Check for updates

Cite this: RSC Adv., 2018, 8, 37098

\title{
Controlled size reduction of rare earth doped nanoparticles for optical quantum technologies
}

\author{
Shuping Liu, ${ }^{\text {a }}$ Diana Serrano, ${ }^{a}$ Alexandre Fossati, ${ }^{a}$ Alexandre Tallaire, ${ }^{a}$ Alban Ferrier ${ }^{\text {ab }}$ \\ and Philippe Goldner (D)*a
}

Rare earth doped nanoparticles with sub-wavelength size can be coupled to optical micro- or nano-cavities to enable efficient single ion readout and control, a key requirement for quantum processors and highfidelity single-ion quantum memories. However, producing small nanoparticles with good dispersion and exploitable optical coherence properties, another key aspect for these applications, is highly challenging by most synthesis and nano-fabrication methods. We report here on the wet chemical etching of $\mathrm{Eu}^{3+}: \mathrm{Y}_{2} \mathrm{O}_{3}$ nanoparticles and demonstrate that a controlled size reduction down to $150 \mathrm{~nm}$, well below the wavelength of interest, $580 \mathrm{~nm}$, can be achieved. The etching mechanism is found to proceed by reaction with grain boundaries and isolated grains, based on obtained particles size, morphology and polycrystalline structure. Furthermore, this method allows maintaining long optical coherence lifetimes $\left(T_{2}\right)$ : the $12.5 \mu \mathrm{s}$ and $9.3 \mu$ s values obtained for $430 \mathrm{~nm}$ initial particles and $150 \mathrm{~nm}$ etched particles respectively, revealing a broadening of only $10 \mathrm{kHz}$ after etching. These values are the longest $T_{2}$ values reported for any nanoparticles, opening the way to new rare-earth based nanoscale quantum technologies.

Received 30th August 2018

Accepted 19th October 2018

DOI: $10.1039 / \mathrm{c} 8 \mathrm{ra0} 07246 \mathrm{a}$

rsc.li/rsc-advances hours of spin coherence lifetime have been reported in $\mathrm{Eu}^{3+}: \mathrm{Y}_{2} \mathrm{SiO}_{5}{ }^{21}$ These attractive properties, unique in the solid state, allow RE doped crystals to be used as quantum lightmatter interfaces, as shown in recent reports on quantum memories and optical to microwave transducers. ${ }^{22-25}$

Despite the striking demonstrations reported in macroscopic crystals, RE nanoparticles offer key advantages. In particular, nanoscale systems can be placed in a high-qualityfactor optical micro- or nano-cavities to take advantage of a stronger light-matter coupling. This can lead to efficient single ion readout, ${ }^{26,27}$ and in the long term, it could give rise to scalable quantum processors and high fidelity memories for quantum networks. ${ }^{19,28,29}$

Nonetheless, obtaining long optical and/or spin coherence lifetimes in nano-materials like nano-diamonds ${ }^{30}$ or RE nanocrystals, ${ }^{31}$ is an outstanding challenge. This is because quantum states are extremely sensitive to even small time-dependent perturbations like fluctuating magnetic fields produced by impurities or defects carrying electron spins. At the nanoscale, such perturbing centres can form on particles surfaces or be introduced by precursors during the synthesis, which ultimately results in reduced coherence lifetimes. Very high quality materials are therefore needed to reach $T_{2}$ values comparable to bulk samples. In this sense, $\mathrm{Eu}^{3+}$ doped $\mathrm{Y}_{2} \mathrm{O}_{3}$ is a particularly interesting candidate: in contrast to other RE materials, ${ }^{27,32-34}$ $\mathrm{Eu}^{3+}$ doped $\mathrm{Y}_{2} \mathrm{O}_{3}$ nanoparticles presenting remarkable optical and spin coherence properties, as well as narrow size distribution and bulk-like optical absorption and emission features can be obtained by chemical synthesis. ${ }^{35-37}$ Our previous studies on
${ }^{a}$ Chimie ParisTech, PSL University, CNRS, Institut de Recherche de Chimie Paris, F-75005 Paris, France. E-mail: shuping.liu@chimieparistech.psl.eu; philippe. goldner@chimieparistech.psl.eu

${ }^{b}$ Sorbonne Université, Faculté des Sciences et Ingénierie, UFR 933, F-75005 Paris, France 
$\mathrm{Eu}^{3+}: \mathrm{Y}_{2} \mathrm{O}_{3}$ nanoparticles synthesized by homogeneous precipitation demonstrated an optical homogeneous linewidth of 45 $\mathrm{kHz}\left(T_{2}=7 \mu \mathrm{s}\right)$ for the $\mathrm{Eu}^{3+}:{ }^{7} \mathrm{~F}_{0} \rightarrow{ }^{5} \mathrm{D}_{0}$ transition at $580 \mathrm{~nm}$ in $400 \mathrm{~nm}$-diameter particles. ${ }^{36}$ Spin homogeneous linewidth down to $40 \mathrm{~Hz}\left(T_{2}=8.1 \mathrm{~ms}\right)$ has also been recently reported. ${ }^{37}$ These results are comparable to values observed in some $\mathrm{Eu}^{3+}: \mathrm{Y}_{2} \mathrm{O}_{3}$ bulk crystals and transparent ceramics. ${ }^{31,38-41}$ Still, optical micro-cavities require low scattering losses in order to reach high quality factor and large cavity-ion coupling. Particles significantly smaller than the optical wavelength, $580 \mathrm{~nm}$ in the case of $\mathrm{Eu}^{3+}$, are therefore necessary, as the scattering losses scale as the sixth power of the particle size. ${ }^{28}$

Here, we propose wet chemical etching as a new approach to obtain well-dispersed and sub-wavelength $\mathrm{RE}$ doped $\mathrm{Y}_{2} \mathrm{O}_{3}$ nanoparticles with narrow homogeneous linewidths. Although the homogeneous precipitation method allows for morphology and size distribution control, the $\mathrm{Y}_{2} \mathrm{O}_{3}$ phase forms after calcination of the precipitated yttrium carbonate precursors $(\mathrm{Y}(\mathrm{OH})$ $\mathrm{CO}_{3} \cdot x \mathrm{H}_{2} \mathrm{O}$, YOC). Former investigations demonstrated that high calcination temperature $\left(\sim 1200{ }^{\circ} \mathrm{C}\right)$ is required to cure defects, achieve sufficient crystalline quality and ensure good optical performance.$^{35,36}$ However, this also leads to an increase in aggregation and sintering of the particles when their size is decreased. Our approach to prevent this consists in synthesising high-quality and well-dispersed $\mathrm{Y}_{2} \mathrm{O}_{3}$ particles with average size in the 400-500 $\mathrm{nm}$ range, and then applying chemical etching to achieve a controlled size reduction. This could allow obtaining particles with appropriate size with respect to the optical micro- or nano-cavity targeted quality factor, while preserving the initial particles optical properties. The etching mechanism and the optical coherence performance at $580 \mathrm{~nm}$ of $\mathrm{Eu}^{3+}: \mathrm{Y}_{2} \mathrm{O}_{3}$ etched particles were analyzed and are here discussed. Homogeneous linewidth broadening as low as $10 \mathrm{kHz}$ was measured for $150 \mathrm{~nm}$ etched nanoparticles starting from $430 \mathrm{~nm}$ initial nanoparticles. The corresponding coherence lifetimes, $9.3 \mu \mathrm{s}$ and $12.5 \mu \mathrm{s}$, are the longest ever reported for any nanoparticles. The results suggest that chemical etching is a promising way to obtain RE doped particles suitable for nanoscale quantum hardware architectures.

\section{Materials and methods}

The initial 0.3 at $\% \mathrm{Eu}^{3+}: \mathrm{Y}_{2} \mathrm{O}_{3}$ nanoparticles were synthesized by homogeneous precipitation with a calcination temperature of $1200^{\circ} \mathrm{C}$. The detailed technological route has been described in previous work. ${ }^{35}$ The etching agent used in the study was glacial acetic acid $\left(\mathrm{CH}_{3} \mathrm{COOH}\right.$, original concentration of $100 \mathrm{wt} \%$, with density $\left.1.05 \mathrm{~g} \mathrm{~cm}^{-3}\right)$. Acetic acid has been previously used as etchant in semiconductor manufacturing processes, with concentrations varying from $20 \mathrm{wt} \%$ to $68 \mathrm{wt} \%{ }^{42}$ The chemical etching experiments were carried out by mixing $\mathrm{Eu}^{3+}: \mathrm{Y}_{2} \mathrm{O}_{3}$ nanoparticles with fresh acid solutions (50 wt\% prepared with deionized water). To ensure homogeneous temperature in the acid solution, etching was done under water-bath, with continuous magnetic agitation. After chemical etching, the nanoparticles were collected by centrifugation and washed several times with deionized water and absolute ethanol to remove the byproducts. The final etched powders were obtained after drying at $80^{\circ} \mathrm{C}$ for $24 \mathrm{~h}$. A post microwave treatment under oxygen plasma was performed to remove possible impurities. In order to clarify the effect of various etching conditions on the structures of the nanoparticles, etching time from 1 to $5 \mathrm{~h}$, water-bath temperature from 40 to $70{ }^{\circ} \mathrm{C}$ and acetic acid concentration from 40 to $70 \mathrm{wt} \%$ were investigated. No dependence on the acetic acid content was found, while the effect of etching time and temperature is later discussed in the manuscript.

The morphology of initial and etched particles was observed by scanning electron microscopy (SEM, Zeiss Leo1530) and transmission electron microscopy (TEM, JEOL-JEM-100CXI) operating at $100 \mathrm{kV}$. The particle size distributions were calculated with Image J software based on at least 300 nanoparticles from different SEM images. X-ray diffraction (XRD) measurements were performed on a Panalytical XPert Pro diffractometer with an incident beam Ge monochromator. Crystallite or single grain sizes were determined from the FWHM of 4 different diffraction peaks by applying the Scherrer equation. Mass losses due to etching were derived from the concentration of yttrium in the etched solutions measured by inductively coupled plasma atomic emission spectrometry (ICP-AES, ThermalFisher icp $6000)^{43,44}$

Inhomogeneous and homogeneous linewidths were measured on several initial and etched particles with different average particle size after etching. The samples in form of powders were placed in a helium bath cryostat (Janis SVT-200) and maintained in a copper holder between two glass plates. The detection was carried out by collecting light scattered through the sample as explained in previous works. ${ }^{31,36}$ Inhomogeneous linewidths $\left(\Gamma_{\mathrm{inh}}\right)$ were recorded, for the $\mathrm{Eu}^{3+}:{ }^{7} \mathrm{~F}_{0} \rightarrow$ ${ }^{5} \mathrm{D}_{0}$ transition, at approximately $10 \mathrm{~K}$ by fluorescence excitation using a CW dye ring laser (Sirah Matisse DS, $200 \mathrm{kHz}$ linewidth) pumped by a Coherent Verdi G10 laser. A long-pass filter (600 nm cut-off wavelength) was placed in front of the detector (APD Thorlabs $110 \mathrm{~A} / \mathrm{M}$ ) to reject the excitation light. Homogeneous linewidths $\left(\Gamma_{\mathrm{h}}\right)$ were determined from coherence lifetimes $\left(T_{2}\right)$, the latter measured by two pulse photon echo experiments at the center of the ${ }^{7} \mathrm{~F}_{0} \rightarrow{ }^{5} \mathrm{D}_{0}(580.88 \mathrm{~nm}$ in vacuum) transition at $1.4 \mathrm{~K}$. The length of the exciting and rephasing pulses in the sequence was 1 and $1.5 \mu$ s respectively.

\section{Results and discussion}

\subsection{Structural characterizations and etching mechanism}

Several series of $\mathrm{Eu}^{3+}$ doped $\mathrm{Y}_{2} \mathrm{O}_{3}$ nanoparticles were synthesized by homogenous precipitation, calcined at $1200{ }^{\circ} \mathrm{C}$, and subsequently etched in acetic acid solutions of $50 \mathrm{wt} \%$ acid content $\left(8.53 \mathrm{~mol} \mathrm{~L}^{-1}\right)$. A weak acid $\left(\mathrm{p} K_{\mathrm{a}}=4.76\right.$ at $\left.25^{\circ} \mathrm{C}\right)$ was chosen for low etching rates, allowing control over the size reduction process. Fig. 1 shows the evolution of the particle size distribution and morphology as a function of etching time. The initial $\mathrm{Eu}^{3+}: \mathrm{Y}_{2} \mathrm{O}_{3}$ nanoparticles exhibit spherical, well-dispersed morphology and particle size of $450 \pm 56 \mathrm{~nm}$ according to lognormal fit. As observed, when etching time increases from 1 to 5 hours, the nanoparticles turn to be gradually smaller and their 

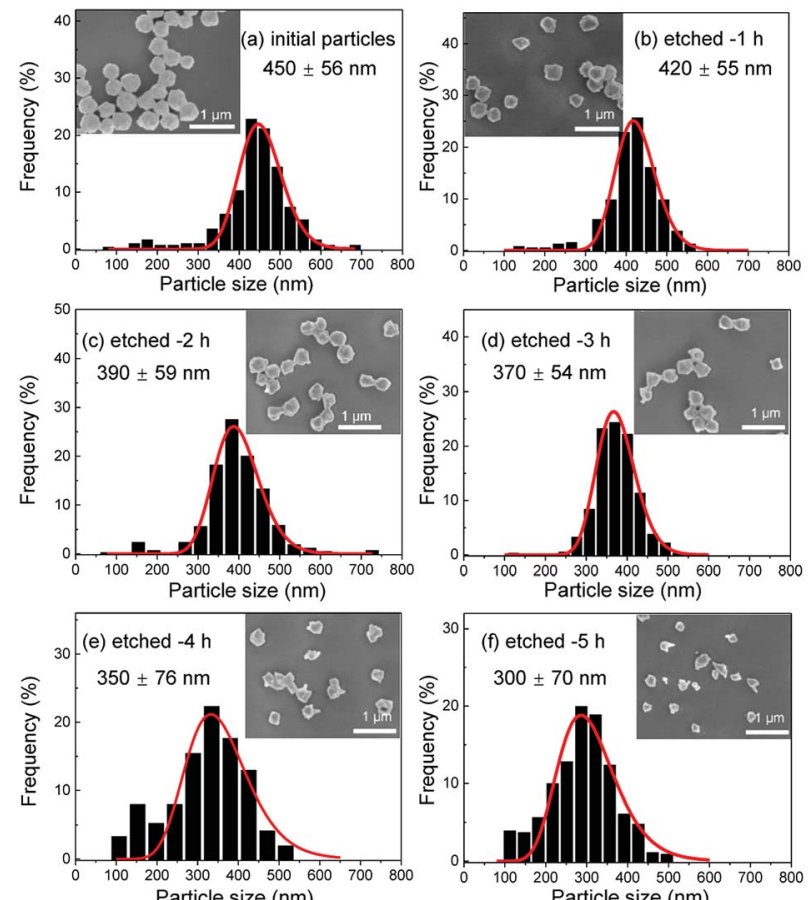

Fig. 1 Scanning electron microscopy (SEM) micrographs and size distributions of the initial and etched $\mathrm{Eu}^{3+}: \mathrm{Y}_{2} \mathrm{O}_{3}$ nanoparticles for etching times increasing from 1 to 5 hours (etching temperature $T=50^{\circ} \mathrm{C}$ ).

shape changes, as evidenced by the appearance of sharp edges and facets. It can be noted that the size distribution of the etched particles stays comparable to that of the initial particles, slightly broadening after 4 hours etching (Fig. 1d and e).

Inductively coupled plasma atomic emission spectroscopy (ICP-AES) was used to determine the mass loss due to etching by measuring the yttrium element concentration in the acid solution after removing the etched particles. In Fig. 2, the ICP-AES results are compared to the expected mass loss based on the

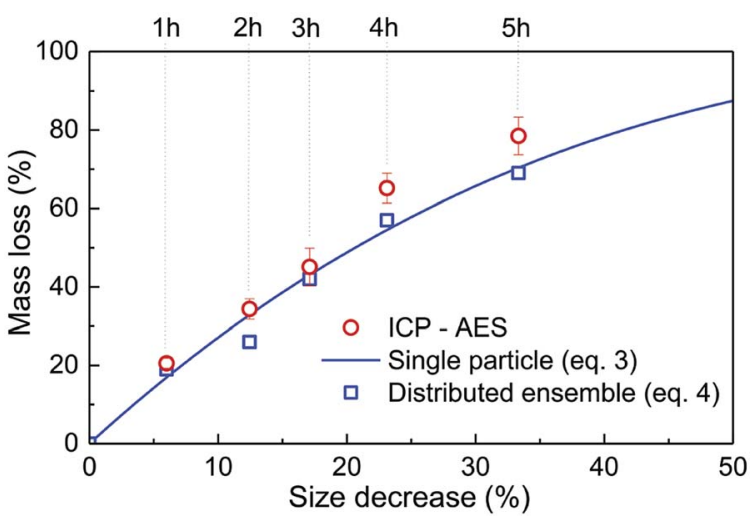

Fig. 2 Relative mass loss due to etching as a function of the relative particle size decrease, $\left(r_{0}-r_{\mathrm{e}}\right) / r_{0}$, with $r_{0}=225 \mathrm{~nm}$ (initial particles) and $r_{\mathrm{e}}$ the average radius of the etched particles after different etching times, e.g. $r_{0}=150 \mathrm{~nm}$ for $t=5 \mathrm{~h}$ (Fig. 1). The experimental ICP-AES results (red dots) are compared to the mass loss expected from the size reduction model (eqn (3) and (4)) for a single average particle (line) and an ensemble of 1000 particles distributed in size as in Fig. 1 (blue squares). progressive size reduction observed in Fig. 1. This simple model assumes that the nanoparticles are perfectly spherical and that the total number of particles remains the same along the etching process. The mass of a single particle is then given by:

$$
m=\rho V=\rho \frac{4}{3} \pi r^{3}
$$

in which $V$ in the particle volume, $r$ the particle radius and $\rho$ the $\mathrm{Y}_{2} \mathrm{O}_{3}$ density. After an etching time $t$, the radius decreases from $r_{0}$ to a specific value $r_{\mathrm{e}}$, and the mass being etched for each particle, $\Delta m$, can be expressed as:

$$
\Delta m=\rho \Delta V_{t}=\rho \frac{4}{3} \pi\left(r_{0}^{3}-r_{\mathrm{e}}^{3}\right)
$$

Then, the relative mass loss per particle just depends on the initial and final particle radii as:

$$
\frac{\Delta m}{m_{0}}=\frac{r_{0}{ }^{3}-r_{\mathrm{e}}{ }^{3}}{r_{0}{ }^{3}}
$$

For an ensemble of particles with total mass $M=\sum_{i} m_{i}$ and a distribution of initial and final radii $r_{0, i}$ and $r_{\mathrm{e}, i}$, eqn (3) becomes:

$$
\frac{\Delta M}{M_{0}}=\frac{\sum_{i}\left(m_{0, i}-m_{\mathrm{e}, i}\right)}{\sum_{i} m_{0, i}}=\frac{\sum_{i}\left(r_{0, i}{ }^{3}-r_{\mathrm{e}, i}{ }^{3}\right)}{\sum_{i} r_{0, i}{ }^{3}}
$$

As displayed in Fig. 2, the mass loss trend measured by ICP-AES is rather well described by eqn (3) and (4), some deviation appearing for long etching times $(>4 \mathrm{~h}$ ). A closer insight into the etched particles inner structure nevertheless indicates that this model, just considering an isotropic and continuous volume reduction of the particles, does not provide an accurate description of the etching mechanism taking place here. Indeed, as the initial $\mathrm{Eu}^{3+}: \mathrm{Y}_{2} \mathrm{O}_{3}$ particles are polycrystalline, they are made of multiple crystalline grains with average sizes ranging from $\approx 100$ to $120 \mathrm{~nm}$. $\mathrm{XRD}$ investigations reveal that the etching process does not lead to a noticeable reduction of the grain size nor increases the grain size dispersion (Fig. 3), in disagreement with the continuous volume reduction hypothesis. To explain this observation, we propose that the acid preferentially reacts with grain boundaries, leading to the detachment of small crystal grains as the etching proceeds, as reported in polycrystalline silicon ultra thin films. ${ }^{45}$ This is supported by TEM images in which nearly detached grains are observed (Fig. 4, red arrows). The mass loss measured by ICP-AES and given in Fig. 2 is mainly attributed to detached grains, which are etched in a rather short time due to their small volume.

Based on the previous conclusions, we redefined the size reduction model by taking into account the polycrystalline nature of the particles, with average grain diameter of $116 \pm$ $8 \mathrm{~nm}$ (Fig. 3b). The mass of a single particle then becomes:

$$
m=n_{\mathrm{g}} \times \rho V_{\mathrm{g}}=n_{\mathrm{g}} \times \rho \frac{4}{3} \pi r_{\mathrm{g}}{ }^{3}
$$


a
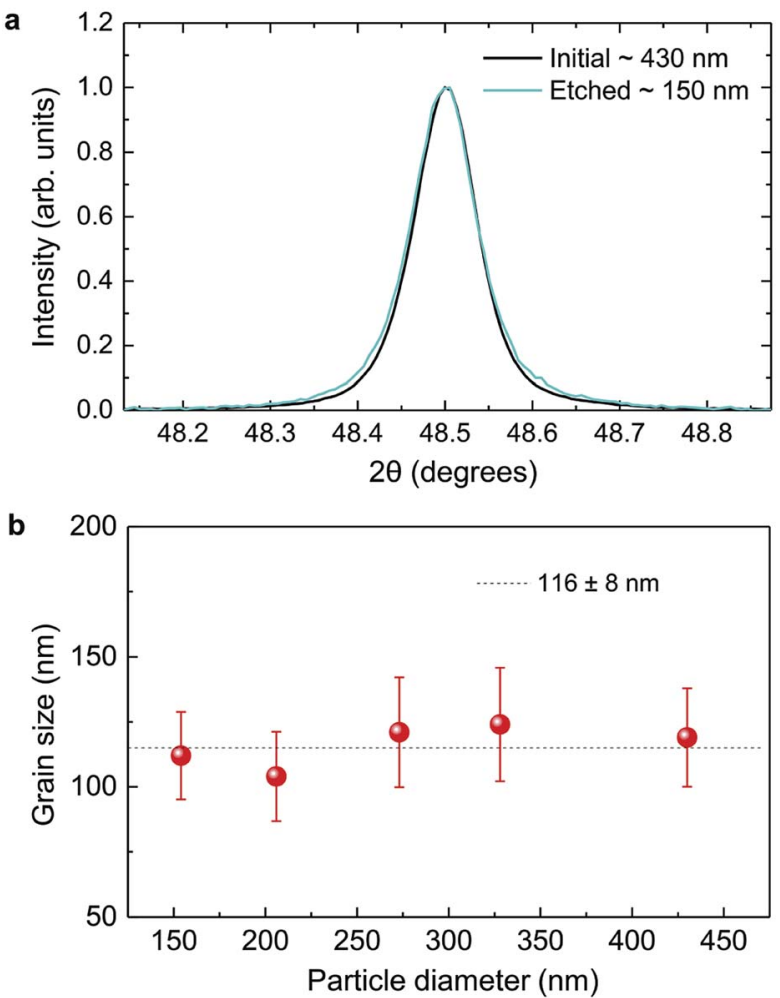

Fig. 3 XRD investigation. (a) Normalized diffraction peak for initial and etched particles with average sizes of 430 and $150 \mathrm{~nm}$ respectively. (b) Grain size as a function of particle size. The dotted line is a guide to the eye.

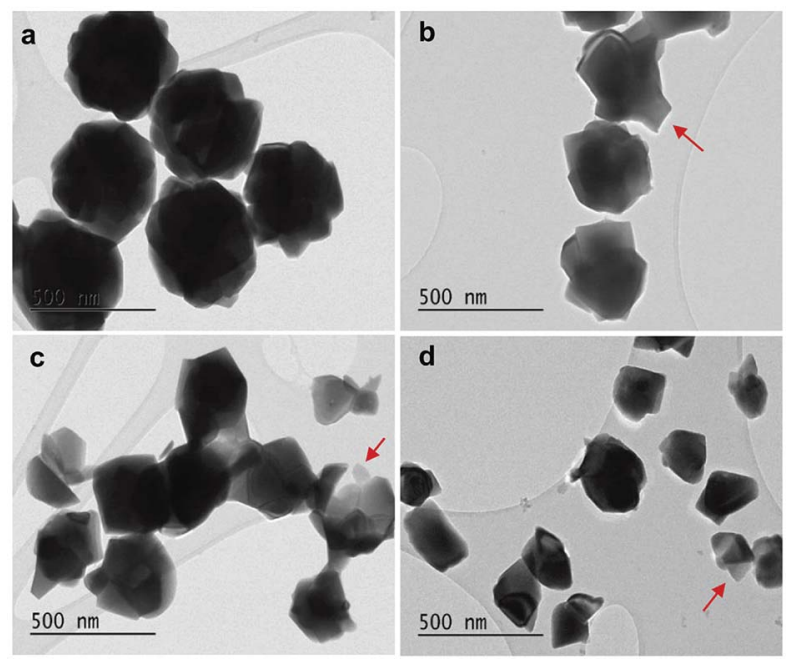

Fig. 4 TEM images of (a) initial $450 \mathrm{~nm}$-diameter particles. Etched particles with final average particle size of (b) $400 \mathrm{~nm}$, (c) $340 \mathrm{~nm}$ and (d) $200 \mathrm{~nm}$ obtained by adjusting etching time and temperature (see Fig. 5). Red arrows: single crystalline grains nearly detached from particles during etching.

in which $V_{\mathrm{g}}$ is the volume of a single crystalline grain, $n_{\mathrm{g}}$ is the number of grains in the particle and $r_{\mathrm{g}}$ the grain radius. The relative mass loss per particle is then given by:

$$
\frac{\Delta m}{m_{0}}=\frac{n_{0}-n_{\mathrm{e}}}{n_{0}}
$$

where $n_{0}$ is the number of grains in the initial particle and $n_{\mathrm{e}}$ the number of grains in the final etched particle. From eqn (6), the number of grains which are lost $\left(n_{0}-n_{\mathrm{e}}\right)$, i.e. detached and then fully etched, can be estimated as a function of etching time from the experimental mass loss measured by ICP-AES (Fig. 2). The result is shown in Fig. 5a. The etching rate appears linear, equal to $\sim 9$ grains $\mathrm{h}^{-1}$, or equivalently, $7.6 \times 10^{6} \mathrm{~nm}^{3} \mathrm{~h}^{-1}$ at $T=$ $50^{\circ} \mathrm{C}$. This result is indeed consistent with several experimental evidences: at the rate found, the initial particles of $\sim 450 \mathrm{~nm}$ should be completely etched after approximately 6.5 hours (Fig. 5b). This was indeed observed since almost no particles could be collected after 6 hours etching. The etching rate found also implies that single crystalline grains are dissolved in less than 7 minutes, therefore contributing to the mass loss shortly after being isolated from the particles.

The temperature dependence of the etching rate, $R(T)$, was also determined from the particle size decrease as a function of etching temperature for a fixed etching time $t$. Thus, $R(T)$ was estimated as:

$$
R(T)=\frac{4}{3} \pi \frac{r_{0}{ }^{3}-r_{\mathrm{e}, T^{3}}}{t}
$$

in which $t$ was set equal to 2 hours, $r_{0}=225 \mathrm{~nm}$ and $r_{\mathrm{e}, T}$ is the average radius of the etched particles obtained with different etching temperatures (Fig. 5c). As displayed in Fig. 5d, $R(T)$ follows the law:

$$
R(T) \propto \mathrm{e}^{-\frac{E_{\mathrm{A}}}{k_{\mathrm{B}} T}}
$$

where $k_{\mathrm{B}}$ is the Boltzmann constant and $E_{\mathrm{A}}$ the activation energy, here found equal to $0.52 \mathrm{eV}$ or $50.2 \mathrm{~kJ} \mathrm{~mol}^{-1}$ (Fig. $5 \mathrm{~d}$ ). This value corresponds to a surface-reaction-limited etching process. ${ }^{46}$

In conclusion, it is demonstrated that a controlled size reduction of the initial $\mathrm{Eu}^{3+}: \mathrm{Y}_{2} \mathrm{O}_{3}$ particles is possible by accurately tuning etching time and temperature. Particles smaller than $150 \mathrm{~nm}$ and even single crystalline particles could in principle be obtained by further optimization of both parameters.

\subsection{High resolution and coherent spectroscopy}

The inhomogeneous broadening $\left(\Gamma_{\mathrm{inh}}\right)$ of initial and etched particles was recorded at low temperature $(\sim 10 \mathrm{~K})$ by monitoring the $\mathrm{Eu}^{3+}{ }^{5} \mathrm{D}_{0}$ emissions while scanning a narrowlinewidth single frequency laser through the ${ }^{7} \mathrm{~F}_{0} \rightarrow{ }^{5} \mathrm{D}_{0}$ transition (see Methods). The initial particles showed $\Gamma_{\text {inh }}$ values around $11 \mathrm{GHz}$, as expected for particles annealed at $1200{ }^{\circ} \mathrm{C} .{ }^{35}$ After etching, a clear broadening was observed in all measured samples, as in the example shown in Fig. 6. Both the initial and etched particles lineshapes could be described by Lorentzian functions. This denotes point defects as the source of inhomogeneous broadening. ${ }^{47}$ In non-etched particles, the inhomogeneous broadening is known to be dominated by the $\mathrm{Eu}^{3+}$ ions themselves, highlighting the low content of defects in these materials. ${ }^{35}$ Substitution of $\mathrm{Y}^{3+}$ by Eu${ }^{3+}$ induces strain in 

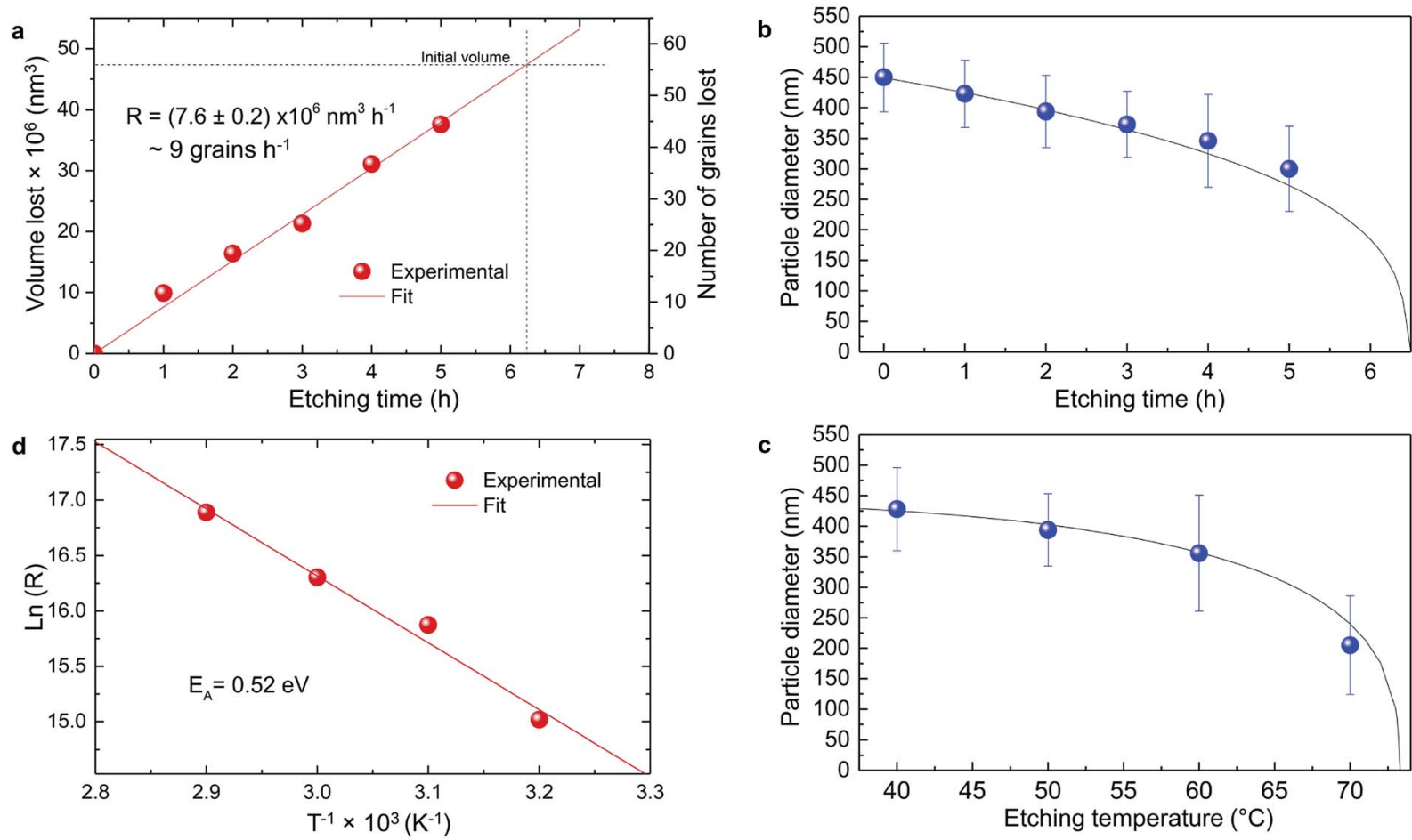

Fig. 5 Particle size evolution with etching time and temperature. (a) Volume lost as a function of etching time and corresponding etching rate. (b) Particle size decrease as a function of etching time with $T=50^{\circ} \mathrm{C}$ (dots) compared to the particle size evolution expected from a rate of $7.6 \times 10^{6}$ $\mathrm{nm}^{3} \mathrm{~h}^{-1}$ (line). (c) Particle size decrease as a function of etching temperature with a fixed etching time of $2 \mathrm{~h}$ (dots) compared to the particle size evolution for an activation energy $E_{\mathrm{A}}=0.52 \mathrm{eV}$ (line). (d) $\ln (R)$ versus $T^{-1}$ showing a linear dependence from which $E_{\mathrm{A}}$ can be estimated according to eqn (8).

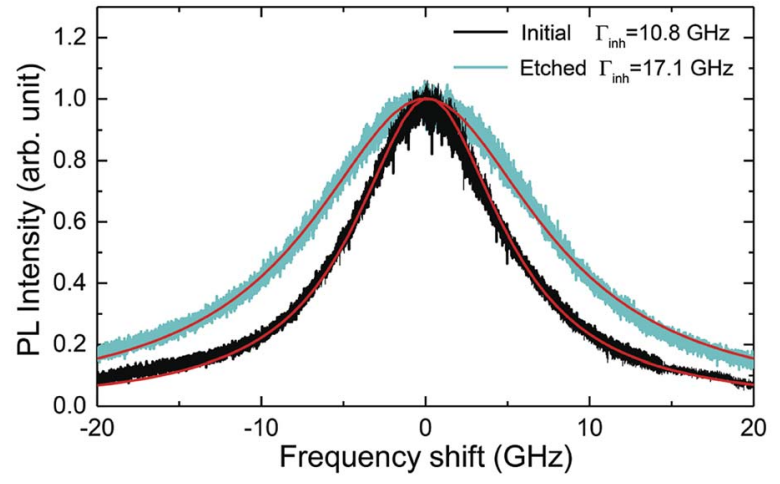

Fig. 6 Inhomogeneous broadening $\left(\Gamma_{\text {inh }}\right)$ of the ${ }^{7} \mathrm{~F}_{0} \rightarrow{ }^{5} \mathrm{D}_{0}$ optical transitions in initial (black) and etched (green) nanoparticles with average sizes of 450 and $200 \mathrm{~nm}$ respectively. Linewidths were estimated by Lorentzian fit.

the crystalline matrix and therefore broadening because of their different atomic radii: $r_{\mathrm{Eu}}=0.950 \AA, r_{\mathrm{Y}}=0.892 \AA{ }^{48}$ Upon etching, strain is not expected to change in the crystalline grains since their size is not noticeably changed during the process, as discussed in the previous section. We therefore attribute the additional broadening observed in etched particles to an increase in point defects at the surface of the crystalline grains, in agreement with the Lorentzian lineshape of the transition.
In order to determine the impact of etching on the optical coherence of the particles, we measured optical coherence lifetimes $\left(T_{2}\right)$ in a series of etched nanoparticles. Starting from an initial size of $430 \mathrm{~nm}$, the smallest particles had an average diameter of $150 \mathrm{~nm}$ after etching. Coherence lifetimes and homogeneous linewidths $\Gamma_{\mathrm{h}}=\left(\pi T_{2}\right)^{-1}$ were obtained from twopulse photon echo measurements ${ }^{31,36}$ at $1.4 \pm 0.1 \mathrm{~K}$ (see Methods). As shown in Fig. 7a, they could be fitted by a single exponential, as previously observed in other nanocrystals. ${ }^{31}$ All measured homogeneous linewidths were below $100 \mathrm{kHz}$ and the additional broadening attributed to etching $\left(\Gamma_{\mathrm{h}, \text { etched }}-\right.$ $\left.\Gamma_{\mathrm{h}, \text { initial }}\right)$ did not show a clear dependence on particle size as displayed in Fig. 7b. In particular, the smallest particles homogeneous linewidth was broadened by only $10 \mathrm{kHz}$ compared to the initial particles. In a previous study, we attributed the main contributions to $\mathrm{Eu}^{3+}$ homogeneous linewidth in nanoparticles to fluctuations due to residual disorder and surface charges. ${ }^{36}$ In all likelihood, the additional broadening induced by etching results from surface modifications (Fig. 4). Since the broadening is only weakly dependent on the particle size, it seems probable that it occurs at the crystalline grain level, whose size does not change with etching (Fig. 3). It could be explained by an increase of fluctuations related to disorder, which increases after etching as evidenced by $\Gamma_{\mathrm{inh}}$ measurements. Surface charges could also increase after etching due for example to the creation of dangling bonds. 

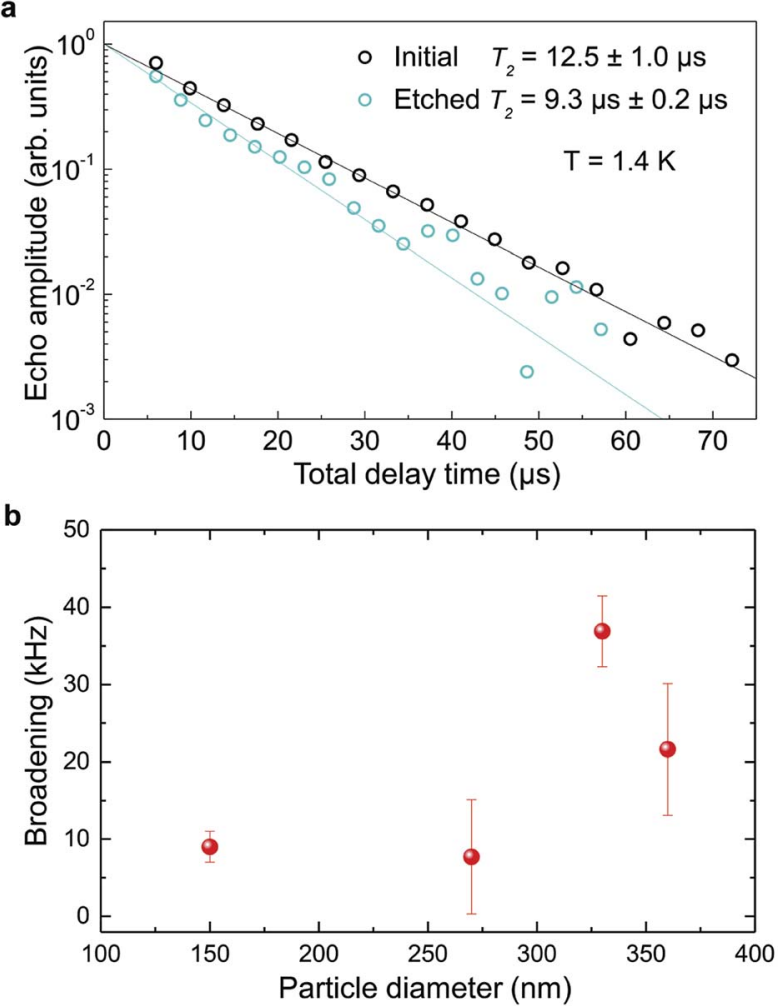

Fig. 7 Optical coherence lifetimes and homogeneous broadening. (a) Photon echo decays for initial and etched nanoparticles at $1.4 \mathrm{~K}$ with average sizes of 430 and $150 \mathrm{~nm}$ respectively. $T_{2}$ values were derived by single exponential fit to the decay data. (b) Additional broadening due to etching as a function of the particles size. Error bars represent \pm 1 standard deviation from several measurements.

Further studies involving temperature dependence of $\Gamma_{\mathrm{h}}$ and 3pulse echoes decays could help clarify this point. ${ }^{36,40}$

These results demonstrate that wet chemical etching can be very useful to reduce particle size while maintaining very narrow homogeneous linewidths. For the smallest particles, we are able to decrease particle size by $65 \%$, from $430 \mathrm{~nm}$ to $150 \mathrm{~nm},(96 \%$ reduction in volume), while obtaining $\Gamma_{\mathrm{h}}=34.2 \mathrm{kHz}$, an unmatched value for any nanoparticle to the best of our knowledge. The significance of this linewidth can be illustrated by comparing it with the interactions used to implement quantum gates in rare earth doped crystals. ${ }^{49}$ Based on $\mathrm{Eu}^{3+}$ optical Stark coefficients, electric dipole-dipole interactions will induce frequency shifts equal to $\Gamma_{\mathrm{h}}$, i.e. $34.2 \mathrm{kHz}$, for ions separated by about $10 \mathrm{~nm} \cdot{ }^{36}$ In the $150 \mathrm{~nm}$ particles, the average distance between $\mathrm{Eu}^{3+}$ ions in the $\mathrm{C}_{2}$ site for $0.3 \%$ doped $\mathrm{Y}_{2} \mathrm{O}_{3}$ particles is $3.2 \mathrm{~nm}$, whereas a single particle contains about $10^{5}$ $\mathrm{Eu}^{3+}$ ions. This suggests that a large fraction of ions could interact strongly enough to explore $\mathrm{Eu}^{3+}-\mathrm{Eu}^{3+}$ interactions and 2-qubit gates. Furthermore, a fibre micro-cavity containing a $150 \mathrm{~nm}$ particle could reach quality factors of a few $1000 \mathrm{~s}$ at $580 \mathrm{~nm}$, an increase of nearly three orders of magnitude compared to $400 \mathrm{~nm}$ particles, which should be high enough to show enhanced light coupling with $\mathrm{Eu}^{3+}$ ions. ${ }^{28}$

\section{Conclusion}

In conclusion, we have found that chemical etching can be used for etching $\mathrm{Eu}^{3+}: \mathrm{Y}_{2} \mathrm{O}_{3}$ oxide polycrystalline nanoparticles at controlled rates. The particles size can be decreased from initial large particles in the $400-500 \mathrm{~nm}$ range to much smaller ones (i.e. $150 \mathrm{~nm}$ ) with a narrow distribution, good dispersion and without obvious changes on the single crystallite size. Based on imaging and structural analysis we propose an etching mechanism that acetic acid tends to open the grain boundaries in the particles, detaching single crystalline grains that are quickly etched, and effectively reducing the size of the remaining particles. Furthermore, we demonstrate that chemical etching has a limited impact on optical performance of the nanoparticles, leading to homogeneous broadenings below $40 \mathrm{kHz}$ for particles between 360 and $150 \mathrm{~nm}$. Moreover, the coherence times of $12.5 \mu \mathrm{s}\left(\Gamma_{\mathrm{h}}=25.5 \mathrm{kHz}\right)$ for $430 \mathrm{~nm}$ initial particles and $9.3 \mu \mathrm{s}\left(\Gamma_{\mathrm{h}}=34.2 \mathrm{kHz}\right)$ for $150 \mathrm{~nm}$ etched nanoparticles observed at $1.4 \mathrm{~K}$, are the longest optical coherence times ever reported for nanoparticles. Our results demonstrate that chemical etching is a promising way to synthesize $\mathrm{RE}: \mathrm{Y}_{2} \mathrm{O}_{3}$ nanoparticles suitable for coupling with optical micro-cavities and with long coherence lifetimes, opening the way to efficient nanoscale quantum memories and processors.

\section{Conflicts of interest}

There are no conflicts to declare.

\section{Acknowledgements}

This project has received funding from the European Union Horizon 2020 research and innovation programme under grant agreement no. 712721 (NanOQTech). We thank Odile Majerus for her assistance in the ICP-AES measurements, Patricia Beaunier for help in the TEM measurements and Thierry Gacoin for useful comments.

\section{Notes and references}

1 M. J. Weber, Handbook on the Physics and Chemistry of Rare Earths, Elsevier, 1979, vol. 4, pp. 275-315.

2 M. J. F. Digonnet, Rare-Earth-Doped Fiber Lasers and Amplifiers, Revised and Expanded, CRC Press, 2001.

3 C. R. Ronda, T. Jüstel and H. Nikol, J. Alloys Compd., 1998, 275-277, 669-676.

4 B. C. Rowan, L. R. Wilson and B. S. Richards, IEEE J. Sel. Top. Quantum Electron., 2008, 14, 1312-1322.

5 C. Bouzigues, T. Gacoin and A. Alexandrou, ACS Nano, 2011, 5, 8488-8505.

6 M. Abdesselem, M. Schoeffel, I. Maurin, R. Ramodiharilafy, G. Autret, O. Clḿent, P.-L. Tharaux, J.-P. Boilot, T. Gacoin, C. Bouzigues and A. Alexandrou, ACS Nano, 2014, 8, 1112611137.

7 G. Sinha and A. Patra, Chem. Phys. Lett., 2009, 473, 151-154. 8 W. J. Kim, M. Nyk and P. N. Prasad, Nanotechnology, 2009, 20, 185301. 
9 C. W. Thiel, T. Böttger and R. L. Cone, J. Lumin., 2011, 131, 353-361.

10 W. Tittel, M. Afzelius, T. Chaneliere, R. L. Cone, S. Kröll, S. A. Moiseev and M. Sellars, Laser Photonics Rev., 2010, 4, 244-267.

11 P. Goldner, A. Ferrier and O. Guillot-Noël, Handb. Phys. Chem. Rare Earths, 2015, 46, 1-78.

12 N. Kunkel and P. Goldner, Z. Anorg. Allg. Chem., 2018, 644, 66-76.

13 S. Welinski, A. Ferrier, M. Afzelius and P. Goldner, Phys. Rev. B, 2016, 94, 155116.

14 R. W. Equall, Y. Sun, R. L. Cone and R. M. Macfarlane, Phys. Rev. Lett., 1994, 72, 2179-2182.

15 T. Böttger, C. W. Thiel, R. L. Cone and Y. Sun, Phys. Rev. B, 2009, 79, 115104.

16 I. Usmani, M. Afzelius, H. de Riedmatten and N. Gisin, Nat. Commun., 2010, 1, 12.

17 R. W. Equall, R. L. Cone and R. M. Macfarlane, Phys. Rev. B, 1995, 52, 3963-3969.

18 A. Louchet, J. S. Habib, V. Crozatier, I. Lorgeré, F. Goldfarb, F. Bretenaker, J.-L. Le Gouët, O. Guillot-Noël and P. Goldner, Phys. Rev. B, 2007, 75, 035131.

19 T. Zhong, J. M. Kindem, J. G. Bartholomew, J. Rochman, I. Craiciu, E. Miyazono, M. Bettinelli, E. Cavalli, V. Verma, S. W. Nam, F. Marsili, M. D. Shaw, A. D. Beyer and A. Faraon, Science, 2017, 357, 1392-1395.

20 T. Böttger, C. W. Thiel, Y. Sun and R. L. Cone, Phys. Rev. B, 2006, 73, 075101.

21 M. Zhong, M. P. Hedges, R. L. Ahlefeldt, J. G. Bartholomew, S. E. Beavan, S. M. Wittig, J. J. Longdell and M. J. Sellars, Nature, 2015, 517, 177.

22 F. Bussières, C. Clausen, A. Tiranov, B. Korzh, V. B. Verma, S. W. Nam, F. Marsili, A. Ferrier, P. Goldner, H. Herrmann, C. Silberhorn, W. Sohler, M. Afzelius and N. Gisin, Nat. Photonics, 2014, 8, 775.

23 E. Saglamyurek, J. Jin, V. B. Verma, M. D. Shaw, F. Marsili, S. W. Nam, D. Oblak and W. Tittel, Nat. Photonics, 2015, 9, 83.

24 N. Maring, P. Farrera, K. Kutluer, M. Mazzera, G. Heinze and H. de Riedmatten, Nature, 2017, 551, 485.

25 L. A. Williamson, Y.-H. Chen and J. J. Longdell, Phys. Rev. Lett., 2014, 113, 203601.

26 R. Kolesov, K. Xia, R. Reuter, R. Stöhr, A. Zappe, J. Meijer, P. R. Hemmer and J. Wrachtrup, Nat. Commun., 2012, 3, 1029.

27 T. Utikal, E. Eichhammer, L. Petersen, A. Renn, S. Götzinger and V. Sandoghdar, Nat. Commun., 2014, 5, 3627.

28 B. Casabone, J. Benedikter, T. Hümmer, F. Beck, K. de Oliveira Lima, T. W. Hänsch, A. Ferrier, P. Goldner, H. de Riedmatten and D. Hunger, New J. Phys., 2018, 20(9), 095006.
29 A. Dibos, M. Raha, C. Phenicie and J. Thompson, arXiv:1711.10368v1, 2017.

30 H. S. Knowles, D. M. Kara and M. Atatüre, Nat. Mater., 2014, 13, 21-25.

31 A. Perrot, P. Goldner, D. Giaume, M. Lovrić, C. Andriamiadamanana, R. R. Gonçalves and A. Ferrier, Phys. Rev. Lett., 2013, 111, 203601.

32 E. Eichhammer, T. Utikal, S. Götzinger and V. Sandoghdar, New J. Phys., 2015, 17, 1-7.

33 T. Lutz, L. Veissier, C. W. Thiel, P. J. T. Woodburn, R. L. Cone, P. E. Barclay and W. Tittel, Sci. Technol. Adv. Mater., 2016, 17, 63-70.

34 T. Lutz, L. Veissier, C. W. Thiel, P. J. T. Woodburn, R. L. Cone, P. E. Barclay and W. Tittel, J. Lumin., 2017, 191, 2-12.

35 K. de Oliveira Lima, R. Rocha Gonçalves, D. Giaume, A. Ferrier and P. Goldner, J. Lumin., 2015, 168, 276-282.

36 J. G. Bartholomew, K. D. Lima, A. Ferrier and P. Goldner, Nano Lett., 2017, 17, 778-787.

37 D. Serrano, J. Karlsson, A. Fossati, A. Ferrier and P. Goldner, Nat. Commun., 2018, 9, 2127.

38 G. P. Flinn, K. W. Jang, J. Ganem, M. L. Jones, R. S. Meltzer and R. M. Macfarlane, Phys. Rev. B, 1994, 49, 5821-5827.

39 A. Ferrier, C. W. Thiel, B. Tumino, M. O. Ramirez, L. E. Bausá, R. L. Cone, A. Ikesue and P. Goldner, Phys. Rev. B, 2013, 87, 041102.

40 N. Kunkel, J. Bartholomew, S. Welinski, A. Ferrier, A. Ikesue and P. Goldner, Phys. Rev. B, 2016, 94, 184301.

41 J. Karlsson, N. Kunkel, A. Ikesue, A. Ferrier and P. Goldner, J. Phys.: Condens. Matter, 2017, 29, 125501.

42 C.-Y. Lee, C. Chang, W.-P. Shih and C.-L. Dai, Thin Solid Films, 2010, 518, 3992-3998.

43 D. Atanassova, V. Stefanova and E. Russeva, Talanta, 1998, 47, 1237-1243.

44 S. L. C. Ferreira, C. F. de Brito, A. F. Dantas, N. M. Lopo de Araujo and A. C. S. Costa, Talanta, 1999, 48, 1173-1177.

45 S.-J. Yoo, J. Lee and H. Shin, Digest of Papers Microprocesses and Nanotechnology 2000. 2000 International Microprocesses and Nanotechnology Conference (IEEE Cat. No.00EX387), 2000, pp. 248-249.

46 S. Tan, R. Boudreau and M. L. Reed, Technical Digest. MEMS 2001. 14th IEEE International Conference on Micro Electro Mechanical Systems (Cat. No.01CH37090), 2001, pp. 139-142. 47 A. M. Stoneham, Rev. Mod. Phys., 1969, 41, 82.

48 R. D. Shannon and C. T. Prewitt, Acta Crystallogr., Sect. B: Struct. Crystallogr. Cryst. Chem., 1969, 25, 925-946.

49 A. Walther, L. Rippe, Y. Yan, J. Karlsson, D. Serrano, A. N. Nilsson, S. Bengtsson and S. Kröll, Phys. Rev. A, 2015, 92, 022319. 